\title{
The Sum and Difference of Two Constant Elasticity of Variance Stochastic Variables
}

\author{
Chi-Fai Lo \\ Department of Physics, Institute of Theoretical Physics, \\ The Chinese University of Hong Kong, Hong Kong, China \\ Email: cflo@phy.cuhk.edu.hk
}

Received August 20, 2013; revised September 20, 2013; accepted September 27, 2013

Copyright (c) 2013 Chi-Fai Lo. This is an open access article distributed under the Creative Commons Attribution License, which permits unrestricted use, distribution, and reproduction in any medium, provided the original work is properly cited.

\begin{abstract}
We have applied the Lie-Trotter operator splitting method to model the dynamics of both the sum and difference of two correlated constant elasticity of variance (CEV) stochastic variables. Within the Lie-Trotter splitting approximation, both the sum and difference are shown to follow a shifted CEV stochastic process, and approximate probability distributions are determined in closed form. Illustrative numerical examples are presented to demonstrate the validity and accuracy of these approximate distributions. These approximate probability distributions can be used to valuate two-asset options, e.g. spread options and basket options, where the CEV variables represent the forward prices of the underlying assets. Moreover, we believe that this new approach can be extended to study the algebraic sum of $N$ CEV variables with potential applications in pricing multi-asset options.
\end{abstract}

Keywords: Constant Elasticity of Variance Stochastic Variables; Probability Distribution Functions; Backward Kolmogorov Equation; Lie-Trotter Splitting Approximation

\section{Introduction}

Recently Lo [1] proposed a new simple approach to tackle the long-standing problem: "Given two correlated lognormal stochastic variables, what is the stochastic dynamics of the sum or difference of the two variables?"; or equivalently, "What is the probability distribution of the sum or difference of two correlated lognormal stochastic variables?" The solution to this problem has wide applications in many fields including financial modelling, actuarial sciences, telecommunications, biosciences and physics [2-15]. By means of the Lie-Trotter operator splitting method [16], Lo showed that both the sum and difference of two correlated lognormal stochastic processes could be approximated by a shifted lognormal stochastic process, and approximate probability distributions were determined in closed form. Unlike previous studies which treat the sum and difference in a separate manner [2-5, 8,13,15,17-27], Lo's method provides a new unified approach to model the dynamics of both the sum and difference of the two stochastic variables. In addition, in terms of the approximate solutions, Lo presented an analytical series expansion of the exact solutions, which can allow us to improve the approximation systematically.
In this communication we extend Lo's approach to study the dynamics of both the sum and difference of two correlated constant elasticity of variance (CEV) stochastic processes. The CEV process was first proposed by Cox and Ross [28] as an alternative to the lognormal stochastic movements of stock prices in the BlackScholes model. The CEV process, which is defined by the stochastic differential equation

$$
\mathrm{d} S=\sigma S^{\beta / 2} \mathrm{~d} Z \text { for } 0 \leq \beta<2
$$

with $\mathrm{dZ}$ being a standard Weiner process, has been receiving much attention because it has the ability to give rise to a volatility skew and to explain the volatility smile [29-41]. In the limiting case $\beta=2$, the CEV model returns to the conventional Black-Scholes model, whilst it is reduced to the Ornstein-Uhlenbeck model in the case of $\beta=0$. By the Lie-Trotter operator splitting method, we show that both the sum and difference of two correlated CEV processes can be modelled by a shifted CEV process. Approximate probability distributions of both the sum and difference are determined in closed form, and illustrative numerical examples are presented to demonstrate the validity and accuracy of these approximate distributions. 


\section{Lie-Trotter Operator Splitting Method}

We consider two CEV stochastic variables $S_{1}$ and $S_{2}$, which are described by the stochastic differential equations:

$$
\mathrm{d} S_{i}=\sigma_{i} S_{i}^{\beta / 2} \mathrm{~d} Z_{i}, i=1,2
$$

for $0 \leq \beta<2$. Here $\mathrm{d} Z_{i}$ denotes a standard Weiner process associated with $S_{i}$, and the two Weiner processes are correlated as $\mathrm{dZ}_{1} \mathrm{~d} Z_{2}=\rho \mathrm{d} t$. Without loss of generality, we also assume that $\sigma_{1}>\sigma_{2}$. The joint probability distribution function $P\left(S_{1}, S_{2}, t ; S_{10}, S_{20}, t_{0}\right)$ of the two correlated CEV variables obeys the backward Kolmogorov equation [42-45]

$$
\left\{\frac{\partial}{\partial t_{0}}+\hat{L}\right\} P\left(S_{1}, S_{2}, t ; S_{10}, S_{20}, t_{0}\right)=0
$$

where

$$
\begin{aligned}
\hat{L}= & \frac{1}{2} \sigma_{1}^{2} S_{10}^{\beta} \frac{\partial^{2}}{\partial S_{10}^{2}}+\rho \sigma_{1} \sigma_{2} S_{10}^{\beta / 2} S_{20}^{\beta / 2} \frac{\partial^{2}}{\partial S_{10} \partial S_{20}} \\
& +\frac{1}{2} \sigma_{2}^{2} S_{20}^{\beta} \frac{\partial^{2}}{\partial S_{20}^{2}},
\end{aligned}
$$

subject to the boundary condition

$$
P\left(S_{1}, S_{2}, t ; S_{10}, S_{20}, t_{0} \rightarrow t\right)=\delta\left(S_{1}-S_{10}\right) \delta\left(S_{2}-S_{20}\right) .
$$

This joint probability distribution function tells us how probable the two CEV variables assume the values $S_{1}$ and $S_{2}$ at time $t>t_{0}$, provided that their values at $t_{0}$ are given by $S_{10}$ and $S_{20}$. Once $P\left(S_{1}, S_{2}, t ; S_{10}, S_{20}, t_{0}\right)$ is found, the probability distribution of the sum or difference, namely $S^{ \pm} \equiv S_{1} \pm S_{2}$, of the two correlated $\mathrm{CEV}$ variables can be obtained by evaluating the integral

$$
\begin{aligned}
& \bar{P}_{ \pm}\left(S^{ \pm}, t ; S_{10}, S_{20}, t_{0}\right) \\
= & \int_{0}^{\infty} \int_{0}^{\infty} \mathrm{d} S_{1} \mathrm{~d} S_{2} P\left(S_{1}, S_{2}, t ; S_{10}, S_{20}, t_{0}\right) \delta\left(S_{1} \pm S_{2}-S^{ \pm}\right) .
\end{aligned}
$$

Unfortunately, the joint probability distribution function is not available in closed form, except for the case of $\rho=0$. Hence, we must resort to numerical methods, e.g. the finite-difference method or Monte Carlo simulation. Nevertheless, the numerically exact solution does not provide any information about the stochastic dynamics of the sum or difference explicitly.

It is observed that the probability distribution of the sum or difference of the two correlated CEV variables, i.e. $\bar{P}_{ \pm}\left(S^{ \pm}, t ; S_{10}, S_{20}, t_{0}\right)$, also satisfies the same backward Kolmogorov equation given in Equation (3), but with a different boundary condition [43]

$$
\bar{P}_{ \pm}\left(S^{ \pm}, t ; S_{10}, S_{20}, t_{0} \rightarrow t\right)=\delta\left(S_{10} \pm S_{20}-S^{ \pm}\right) .
$$

To solve for $\bar{P}_{ \pm}\left(S^{ \pm}, t ; S_{10}, S_{20}, t_{0}\right)$, we first rewrite the backward Kolmogorov equation in terms of the new variables $S_{0}^{ \pm} \equiv S_{10} \pm S_{20}$ as

$$
\left\{\frac{\partial}{\partial t_{0}}+\hat{L}_{+}+\hat{L}_{0}+\hat{L}_{-}\right\} \bar{P}_{ \pm}\left(S^{ \pm}, t ; S_{0}^{+}, S_{0}^{-}, t_{0}\right)=0
$$

where

$$
\begin{gathered}
\hat{L}_{+}=\frac{1}{2}\left(\frac{S_{0}^{+}}{2}\right)^{\beta}\left\{\sigma_{1}^{2}\left(1+\frac{S_{0}^{-}}{S_{0}^{+}}\right)^{\beta}+2 \rho \sigma_{1} \sigma_{2}\left[1-\left(\frac{S_{0}^{-}}{S_{0}^{+}}\right)^{2}\right]^{\beta / 2}\right. \\
\left.+\sigma_{2}^{2}\left(1-\frac{S_{0}^{-}}{S_{0}^{+}}\right)^{\beta}\right\} \frac{\partial^{2}}{\partial S_{0}^{+2}} \\
\hat{L}_{-}=\frac{1}{2}\left(\frac{S_{0}^{+}}{2}\right)^{\beta}\left\{\begin{array}{l}
\sigma_{1}^{2}\left(1+\frac{S_{0}^{-}}{S_{0}^{+}}\right)^{\beta}-2 \rho \sigma_{1} \sigma_{2} \\
\left.\left.\left.+\sigma_{2}^{2}\left(1-\frac{S_{0}^{-}}{S_{0}^{+}}\right)^{\beta}\right\} \frac{\partial^{2}}{\partial S_{0}^{-2}}\right)^{2}\right]^{\beta / 2}
\end{array}\right. \\
\left.\hat{L}_{0}=\left\{\begin{array}{c}
\sigma_{1}^{2}\left(\frac{S_{0}^{+}}{+}+S_{0}^{-}\right. \\
2
\end{array}\right)^{\beta}-\sigma_{2}^{2}\left(\frac{S_{0}^{+}-S_{0}^{-}}{2}\right)^{\beta}\right\} \frac{\partial^{2}}{\partial S_{0}^{+} \partial S_{0}^{-}} \quad(11) \\
\sigma_{ \pm}
\end{gathered}
$$

The corresponding boundary condition now becomes

$$
\bar{P}_{ \pm}\left(S^{ \pm}, t ; S_{0}^{+}, S_{0}^{-}, t_{0} \rightarrow t\right)=\delta\left(S_{0}^{ \pm}-S^{ \pm}\right) .
$$

Accordingly, the formal solution of Equation (8) is readily given by

$$
\begin{aligned}
& \bar{P}_{ \pm}\left(S^{ \pm}, t ; S_{0}^{+}, S_{0}^{-}, t_{0}\right) \\
= & \exp \left\{\left(t-t_{0}\right)\left(\hat{L}_{+}+\hat{L}_{0}+\hat{L}_{-}\right)\right\} \delta\left(S_{0}^{ \pm}-S^{ \pm}\right) .
\end{aligned}
$$

Since the exponential operator $\exp \left\{\left(t-t_{0}\right)\left(\hat{L}_{+}+\hat{L}_{0}+\hat{L}_{-}\right)\right\}$is difficult to evaluate, we apply the Lie-Trotter operator splitting $\operatorname{method}^{1}$ to ap-

*Suppose that one needs to exponentiate an operator $\hat{C}$ which can be split into two different parts, namely $\hat{A}$ and $\hat{B}$. For simplicity, let us assume that $\hat{C}=\hat{A}+\hat{B}$, where the exponential operator $\exp (\hat{C})$ is difficult to evaluate but $\exp (\hat{A})$ and $\exp (\hat{B})$ are either solvable or easy to deal with. Under such circumstances the exponential operator $\exp (\varepsilon \hat{C})$, with $\varepsilon$ being a small parameter, can be approximated by the Lie-Trotter splitting formula (Trotter, 1959):

$$
\exp (\varepsilon \hat{C})=\exp (\varepsilon \hat{A}) \exp (\varepsilon \hat{B})+O\left(\varepsilon^{2}\right) .
$$

This can be seen as the approximation to the solution at $t=\varepsilon$ of the equation $\mathrm{d} \hat{Y} / \mathrm{d} t=(\hat{A}+\hat{B}) \hat{Y}$ by a composition of the exact solutions of the equations $\mathrm{d} \hat{Y} / \mathrm{d} t=\hat{A} \hat{Y}$ and $\mathrm{d} \hat{Y} / \mathrm{d} t=\hat{B} \hat{Y}$ at time $t=\varepsilon$. 
proximate the operator by [16,46-49]

$$
\hat{O}_{ \pm}^{L T}=\exp \left\{\left(t-t_{0}\right) \hat{L}_{ \pm}\right\} \exp \left\{\left(t-t_{0}\right)\left(\hat{L}_{0}+\hat{L}_{\mp}\right)\right\},
$$

and obtain an approximation to the formal solution $\bar{P}_{ \pm}\left(S^{ \pm}, t ; S_{0}^{+}, S_{0}^{-}, t_{0}\right)$, namely

$$
\begin{aligned}
& \bar{P}_{ \pm}^{L T}\left(S^{ \pm}, t ; S_{0}^{+}, S_{0}^{-}, t_{0}\right) \\
= & \hat{O}_{ \pm}^{L T} \delta\left(S_{0}^{ \pm}-S^{ \pm}\right)=\exp \left\{\left(t-t_{0}\right) \hat{L}_{ \pm}\right\} \delta\left(S_{0}^{ \pm}-S^{ \pm}\right)
\end{aligned}
$$

where the relation

$$
\exp \left\{\left(t-t_{0}\right)\left(\hat{L}_{0}+\hat{L}_{\bar{\mp}}\right)\right\} \delta\left(S_{0}^{ \pm}-S^{ \pm}\right)=\delta\left(S_{0}^{ \pm}-S^{ \pm}\right) \text {is util- }
$$

ized. For $\left(S_{0}^{-} / S_{0}^{+}\right)^{2} \ll 1$, which is normally valid unless $S_{10}$ and $S_{20}$ are both close to zero, the operators $\hat{L}_{+}$ and $\hat{L}_{-}$can be approximately expressed as

$$
\hat{L}_{ \pm} \cong \frac{1}{2} \tilde{\sigma}_{ \pm}^{2} \tilde{S}_{0}^{ \pm \beta} \frac{\partial^{2}}{\partial \tilde{S}_{0}^{ \pm 2}}
$$

in terms of the two new variables:

$$
\begin{gathered}
\tilde{S}_{0}^{+}=S_{0}^{+}+\left(\frac{\sigma_{1}^{2}-\sigma_{2}^{2}}{\sigma_{+}^{2}}\right) S_{0}^{-} \\
\tilde{S}_{0}^{-}=S_{0}^{-}+\left(\frac{\sigma_{-}^{2}}{\sigma_{1}^{2}-\sigma_{2}^{2}}\right) S_{0}^{+} .
\end{gathered}
$$

Here the parameters $\tilde{\sigma}_{+}$and $\tilde{\sigma}_{-}$are defined by

$$
\begin{gathered}
\tilde{\sigma}_{+}=\frac{\sigma_{+}}{2^{\beta / 2}} \\
\tilde{\sigma}_{-}=\sigma_{-}^{1-\beta}\left(\frac{\sigma_{1}^{2}-\sigma_{2}^{2}}{2}\right)^{\beta / 2} .
\end{gathered}
$$

Obviously, both $\tilde{S}^{+}$and $\tilde{S}^{-}$are CEV random variables defined by the stochastic differential equations

$$
\mathrm{d} \tilde{S}^{ \pm}=\tilde{\sigma}_{ \pm} \tilde{S}^{ \pm \beta / 2} \mathrm{~d} Z_{ \pm}
$$

and their closed-form probability distribution functions are given by

$$
\begin{aligned}
& f^{\mathrm{CEV}}\left(\tilde{S}^{ \pm}, t ; \tilde{S}_{0}^{ \pm}, t_{0}\right) \\
= & \frac{2\left(\tilde{S}_{0}^{ \pm}\right)^{1 / 2}\left(\tilde{S}^{ \pm}\right)^{(1-2 \beta) / 2}}{(2-\beta) \tilde{\sigma}_{ \pm}^{2}\left(t-t_{0}\right)} I_{1 /(2-\beta)}\left(\frac{4\left(\tilde{S}_{0}^{ \pm} \tilde{S}^{ \pm}\right)^{(2-\beta) / 2}}{(2-\beta)^{2} \tilde{\sigma}_{ \pm}^{2}\left(t-t_{0}\right)}\right) \\
& \times \exp \left\{-\frac{2\left[\left(\tilde{S}_{0}^{ \pm}\right)^{2-\beta}+\left(\tilde{S}^{ \pm}\right)^{2-\beta}\right]}{(2-\beta)^{2} \tilde{\sigma}_{ \pm}^{2}\left(t-t_{0}\right)}\right\}
\end{aligned}
$$

for $t>t_{0}$, where $I_{v}(\cdot)$ denotes the modified Bessel function of order $v$. As a result, it can be inferred that within the Lie-Trotter splitting approximation both $S^{+}$ and $S^{-}$are governed by a shifted CEV process. It should be noted that for the Lie-Trotter splitting approximation to be valid, $\tilde{\sigma}_{ \pm}^{2}\left(t-t_{0}\right)$ needs to be small.

\section{Illustrative Numerical Examples}

In Figure 1 we plot the approximate closed-form probability distribution function of the sum $S^{+}$of two uncorrelated CEV variables (i.e. $\rho=0$ ) given in Equation (23) for different values of the input parameters. We start with $S_{10}=110, S_{20}=105, \sigma_{1}=0.5$ and $\sigma_{2}=0.3$ in Figure 1(a). Then, in order to examine the effect of $S_{20}$, we decrease its value to 85 in Figure 1(b) and to 65 in Figure 1(c). In Figures 1(d)-(f) we repeat the same investigation with a new set of values for $\sigma_{1}$ and $\sigma_{2}$, namely $\sigma_{1}=0.3$ and $\sigma_{2}=0.2$. Without loss of generality, we set $t-t_{0}=1$ for simplicity. The (numerically) exact results which are obtained by numerical integrations are also included for comparison. It is clear that the proposed approximation can provide accurate estimates for the exact values.

In order to have a clearer picture of the accuracy, we plot the corresponding errors of the estimation in Figure 2. We can easily see that major discrepancies appear around the peak of the probability distribution function and that the estimation deteriorates as the elasticity parameter $\beta$ increases. It should be noted that the proposed approximation is exact in the special case of $\beta=0$, i.e. the Ornstein-Uhlenbeck model. We also observed that the errors increase with the ratio $S_{0}^{-} / S_{0}^{+}$ and $\tilde{\sigma}_{+}^{2}$ (or equivalently, $\sigma_{1}$ and $\sigma_{2}$ ) as expected.

Next, we apply the same sequence of analysis to the approximate closed-form probability distribution function of the difference $S^{-}$given in Equation (23). Similar observations about the accuracy of the proposed approximation can be made for the difference $S^{-}$, too (see Figures 3 and 4).

\section{Conclusion}

In this communication we have applied a new unified approach proposed by Lo (2012) to model the dynamics of both the sum and difference of two correlated CEV stochastic variables. By the Lie-Trotter operator splitting method, both the sum and difference are shown to follow a shifted CEV stochastic process, and approximate probability distributions are determined in closed form. Illustrative numerical examples are presented to demonstrate the validity and accuracy of these approximate distributions. These approximate probability distributions can be used to valuate two-asset options, e.g. the spread options, where the CEV variables represent the forward prices of the underlying assets. Moreover, we believe that this new approach can be extended to study the 


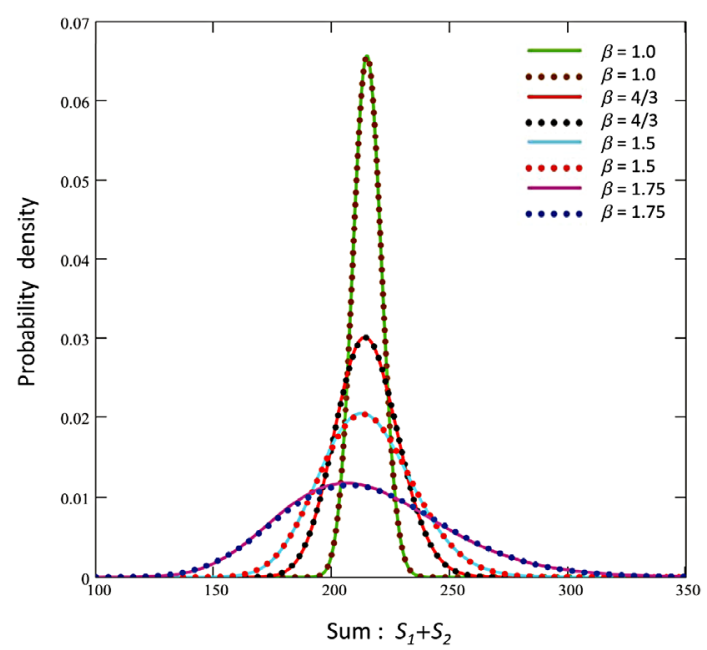

(a)

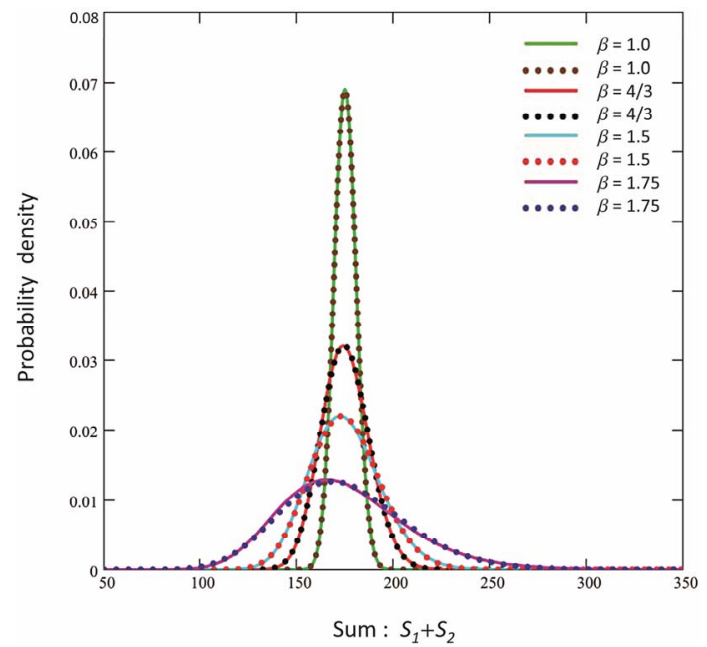

(c)

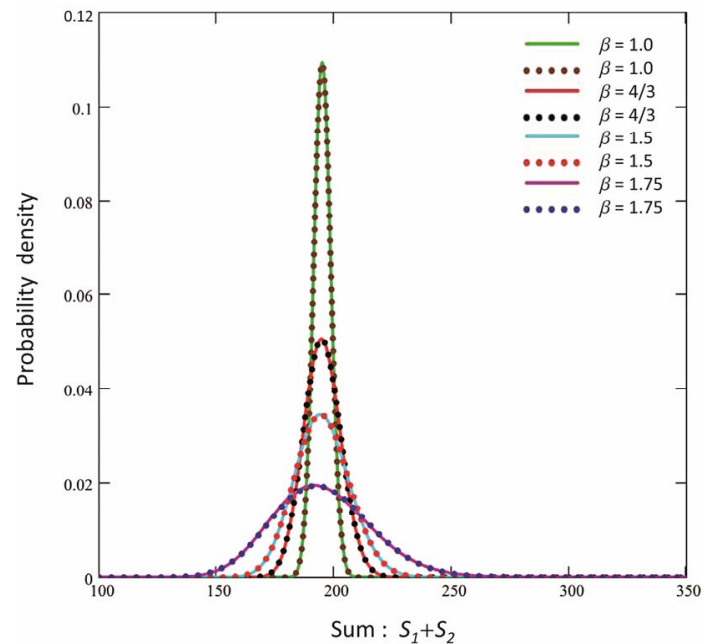

(e)

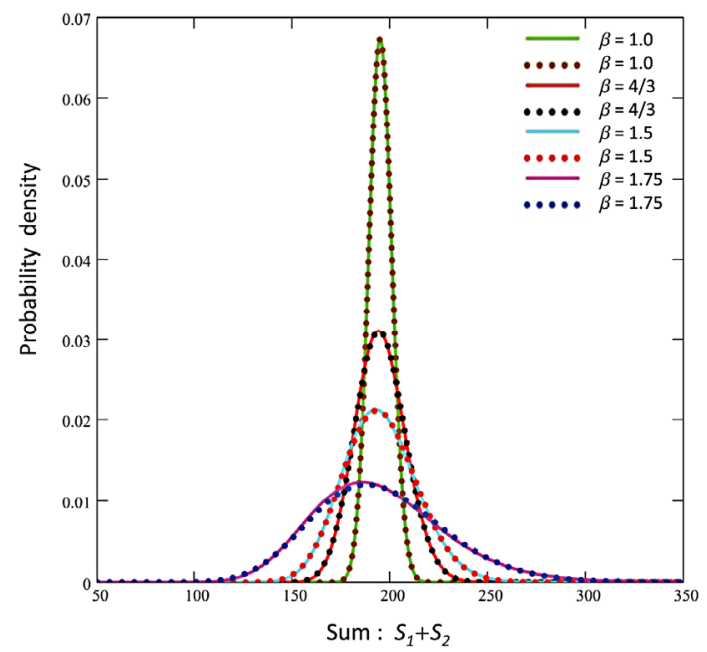

(b)

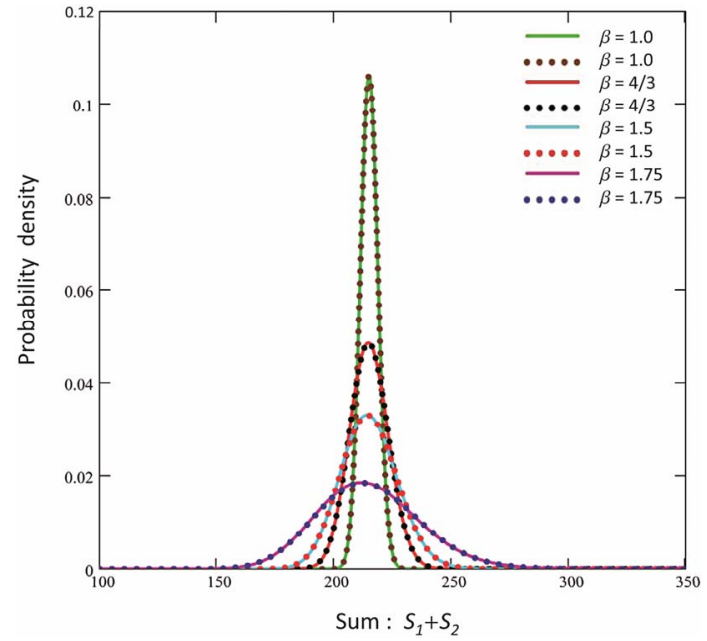

(d)

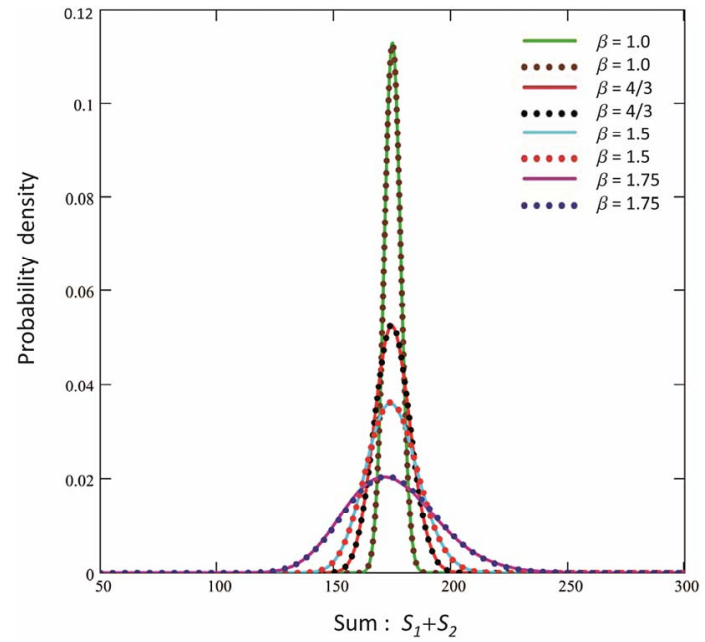

(f)

Figure 1. Probability density vs. $S_{1}+S_{2}$ : the dotted lines denote the distributions of the approximate shifted CEV process, and the solid lines show the exact results. (a) $S_{10}=110, S_{20}=105, \sigma_{1}=0.5$ and $\sigma_{2}=0.3$; (b) $S_{10}=110, S_{20}=85, \sigma_{1}=0.5$ and $\sigma_{2}=0.3$; (c) $S_{10}=110, S_{20}=65, \sigma_{1}=0.5$ and $\sigma_{2}=0.3 ;$ (d) $S_{10}=110, S_{20}=105, \sigma_{1}=0.3$ and $\sigma_{2}=0.2 ;$ (e) $S_{10}=110, S_{20}=85, \sigma_{1}=0.3$ and $\sigma_{2}$ $=0.2 ;$ (f) $S_{10}=110, S_{20}=65, \sigma_{1}=0.3$ and $\sigma_{2}=0.2$. 


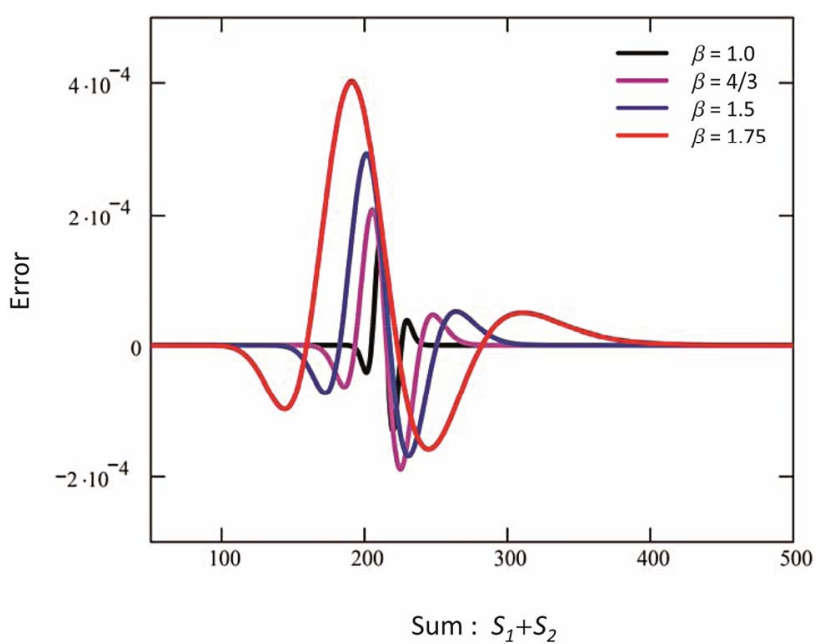

(a)

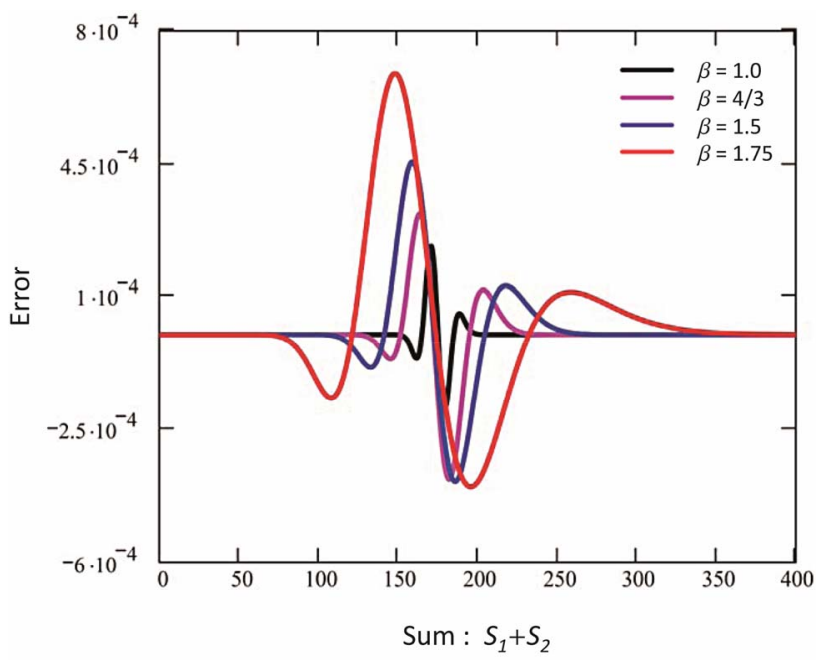

(c)

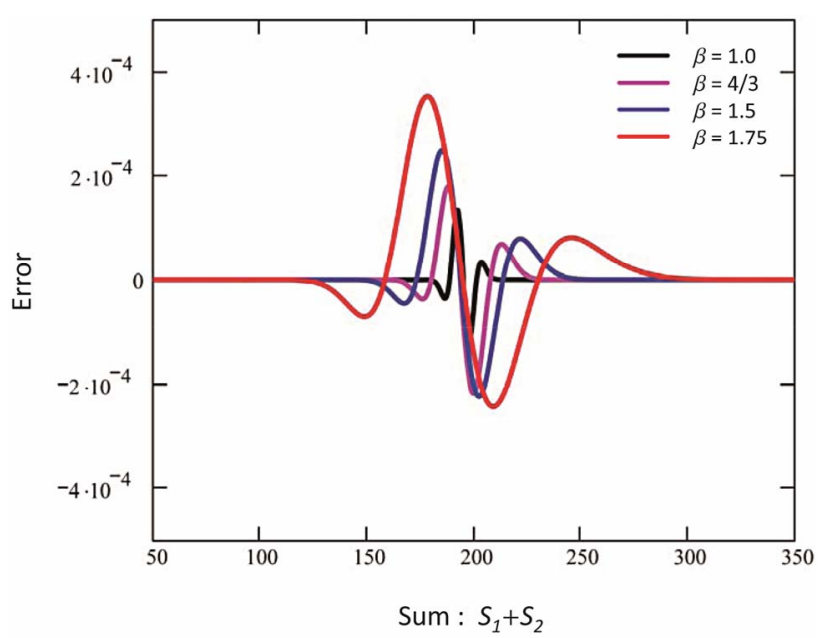

(e)

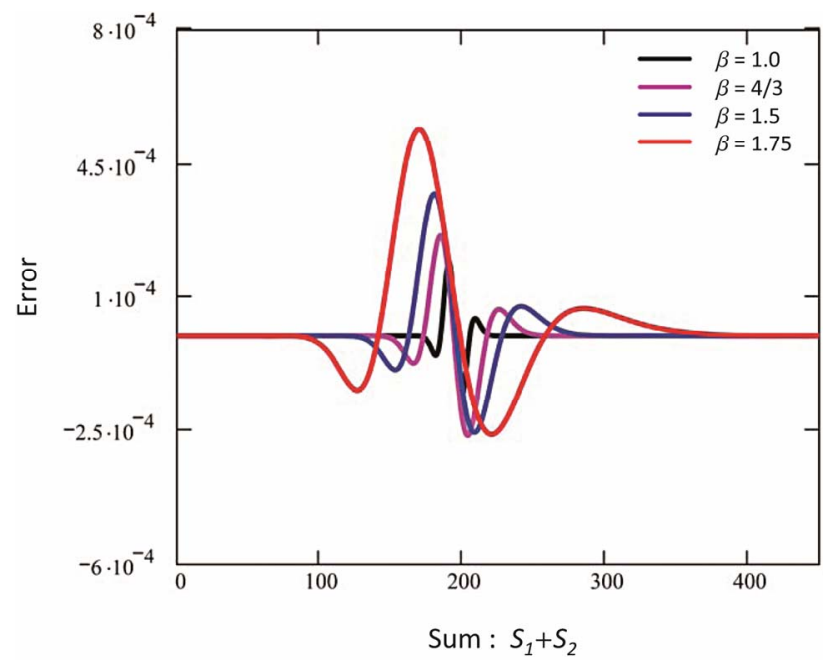

(b)

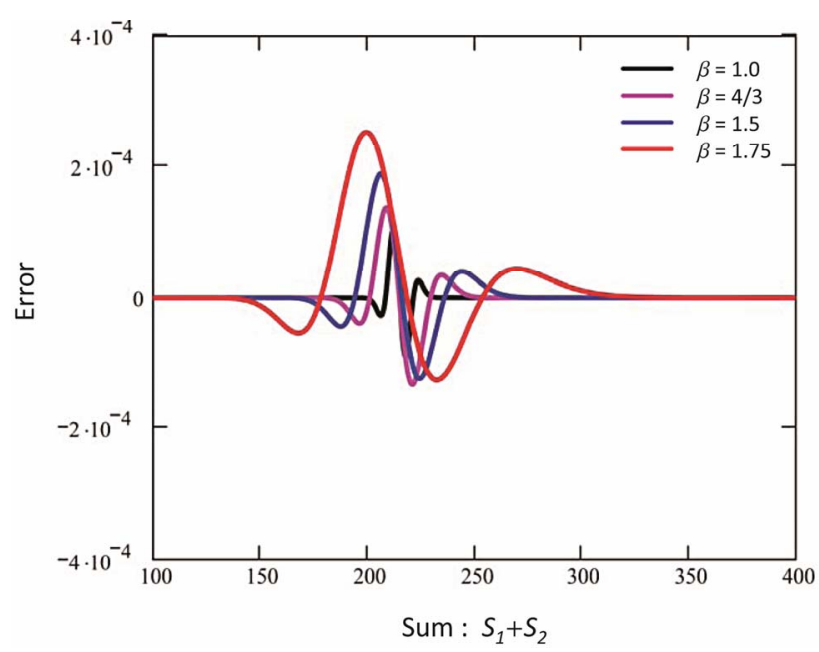

(d)

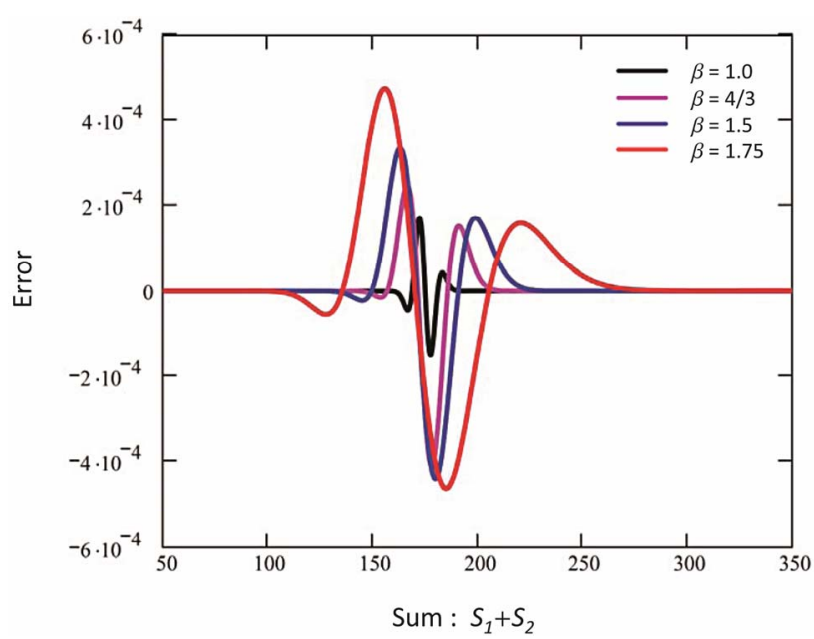

(f)

Figure 2. Error vs. $S_{1}+S_{2}$ : the error is calculated by subtracting the approximate estimate from the exact result. (a) $S_{10}=$ $110, S_{20}=105, \sigma_{1}=0.5$ and $\sigma_{2}=0.3 ;$ (b) $S_{10}=110, S_{20}=85, \sigma_{1}=0.5$ and $\sigma_{2}=0.3$; (c) $S_{10}=110, S_{20}=65, \sigma_{1}=0.5$ and $\sigma_{2}=0.3 ;$ (d) $S_{10}=110, S_{20}=105, \sigma_{1}=0.3$ and $\sigma_{2}=0.2$; (e) $S_{10}=110, S_{20}=85, \sigma_{1}=0.3$ and $\sigma_{2}=0.2$; (f) $S_{10}=110, S_{20}=65, \sigma_{1}=0.3$ and $\sigma_{2}=$ 0.2. 


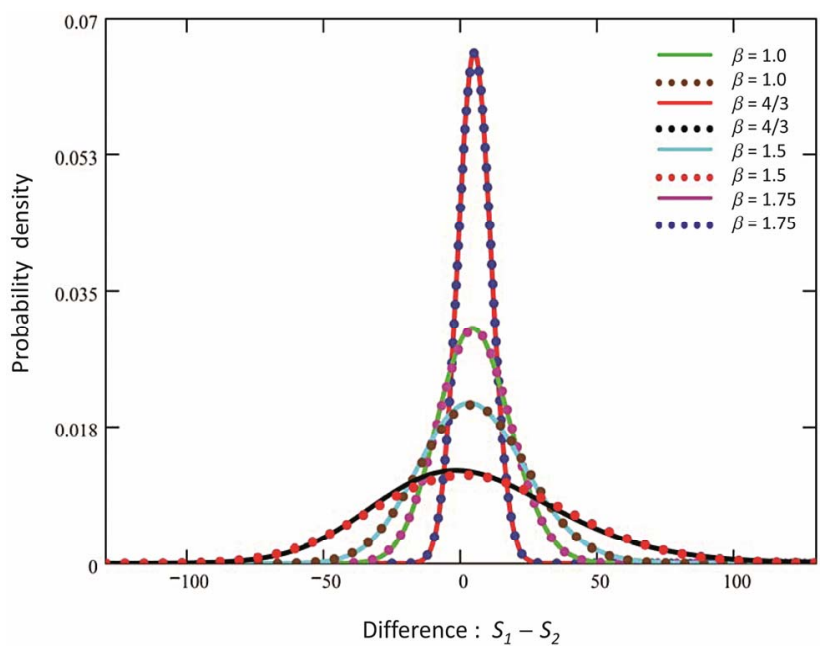

(a)

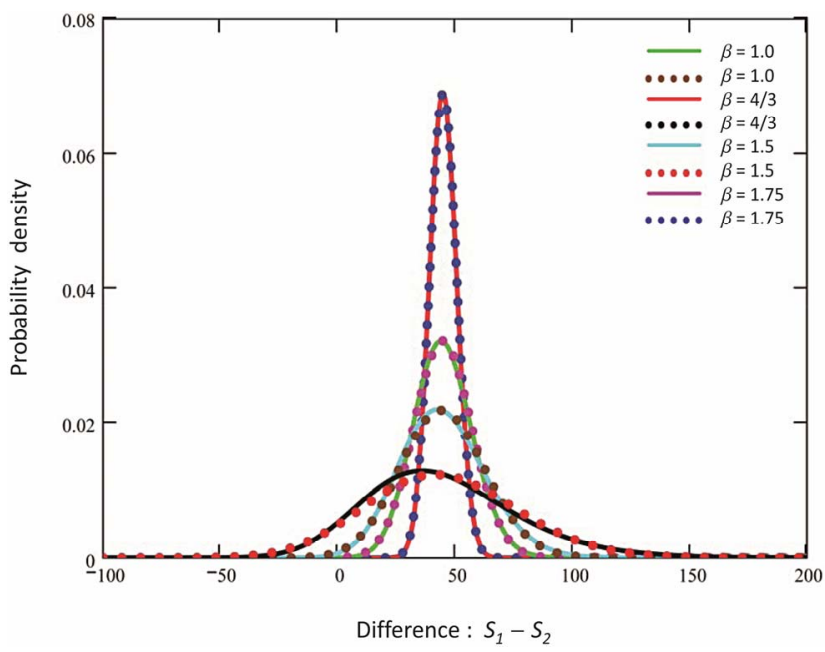

(c)

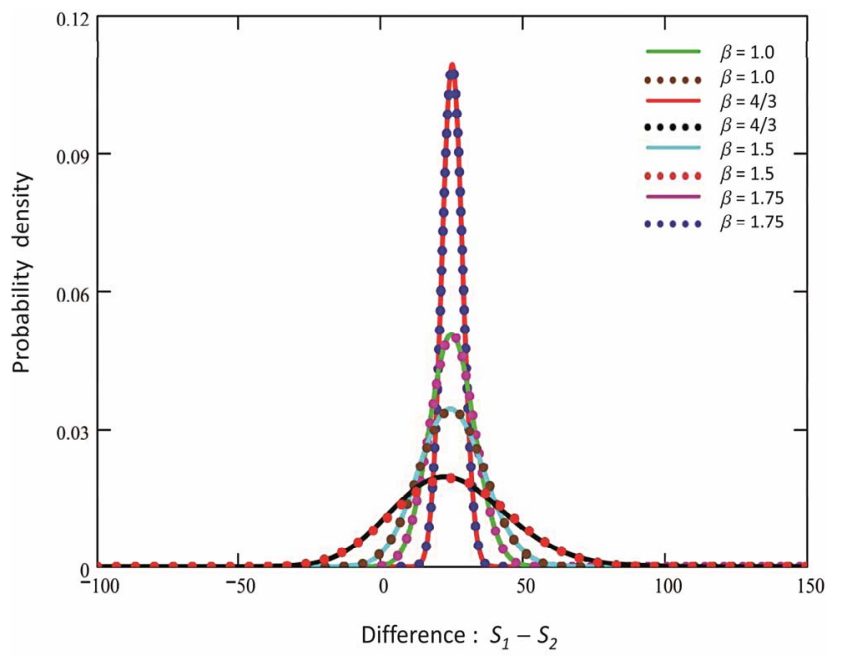

(e)

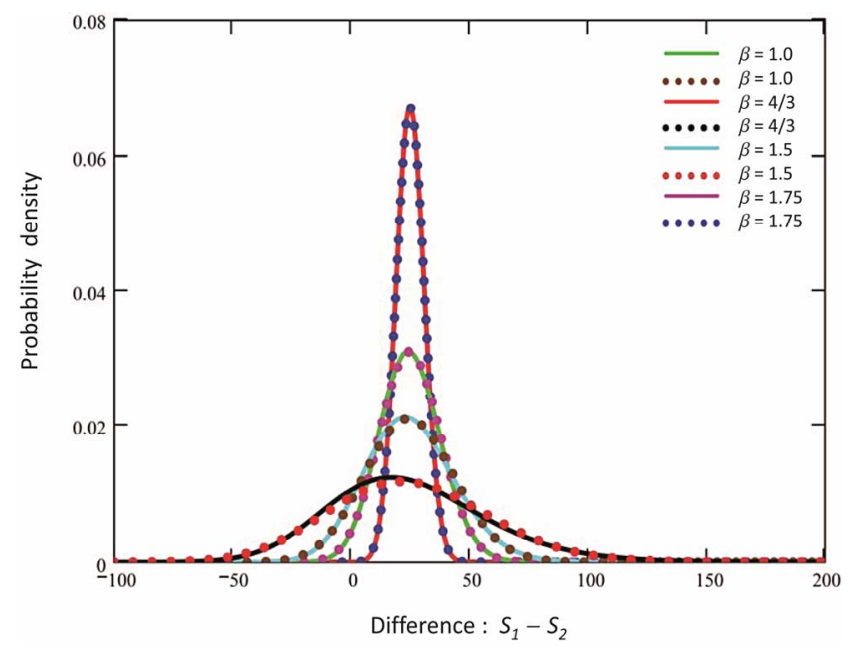

(b)

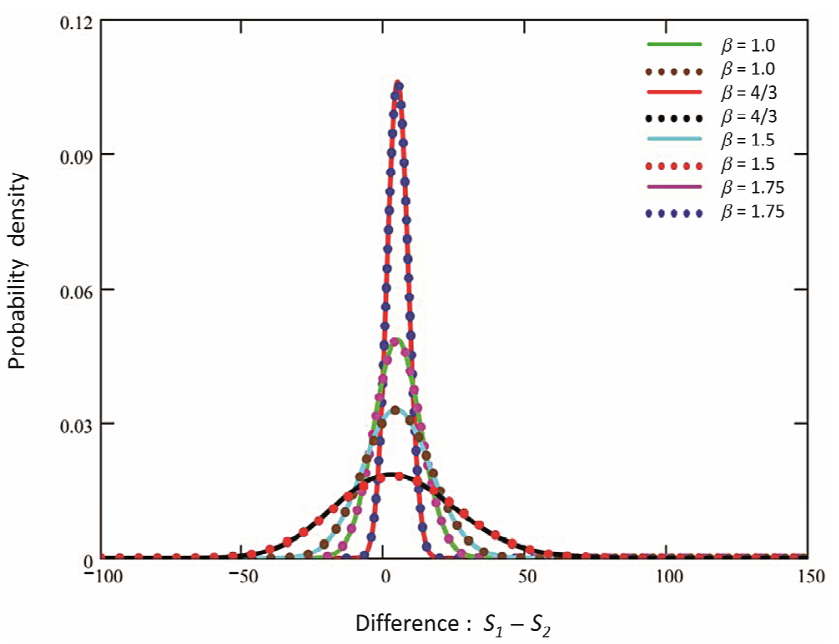

(d)

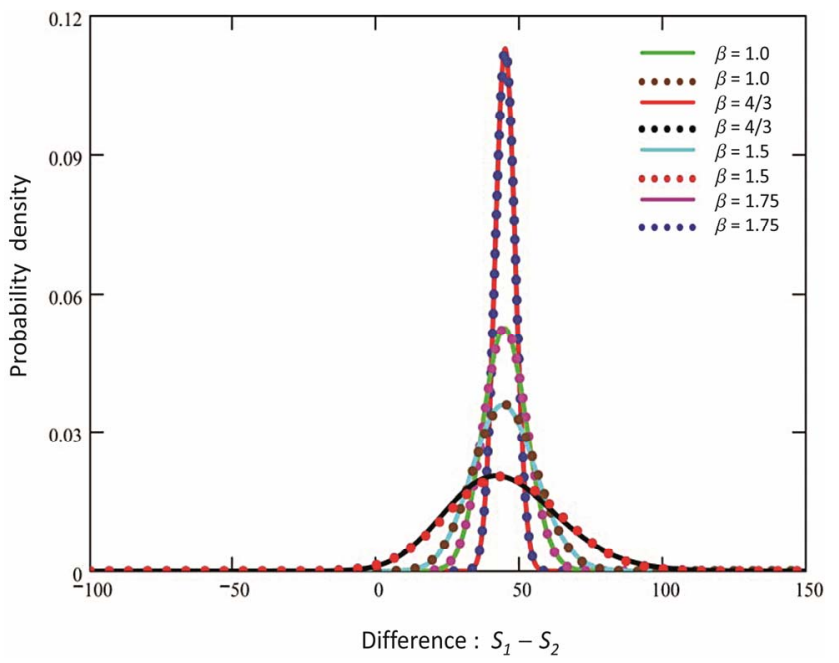

(f)

Figure 3. Probability density vs. $S_{1}-S_{2}$ : the dotted lines denote the distributions of the approximate shifted CEV process, and the solid lines show the exact results. (a) $S_{10}=110, S_{20}=105, \sigma_{1}=0.5$ and $\sigma_{2}=0.3$; (b) $S_{10}=110, S_{20}=85, \sigma_{1}=0.5$ and $\sigma_{2}=0.3$; (c) $S_{10}=110, S_{20}=65, \sigma_{1}=0.5$ and $\sigma_{2}=0.3$; (d) $S_{10}=110, S_{20}=105, \sigma_{1}=0.3$ and $\sigma_{2}=0.2$; (e) $S_{10}=110, S_{20}=85, \sigma_{1}=0.3$ and $\sigma_{2}$ $=0.2$; (f) $S_{10}=110, S_{20}=65, \sigma_{1}=0.3$ and $\sigma_{2}=0.2$. 


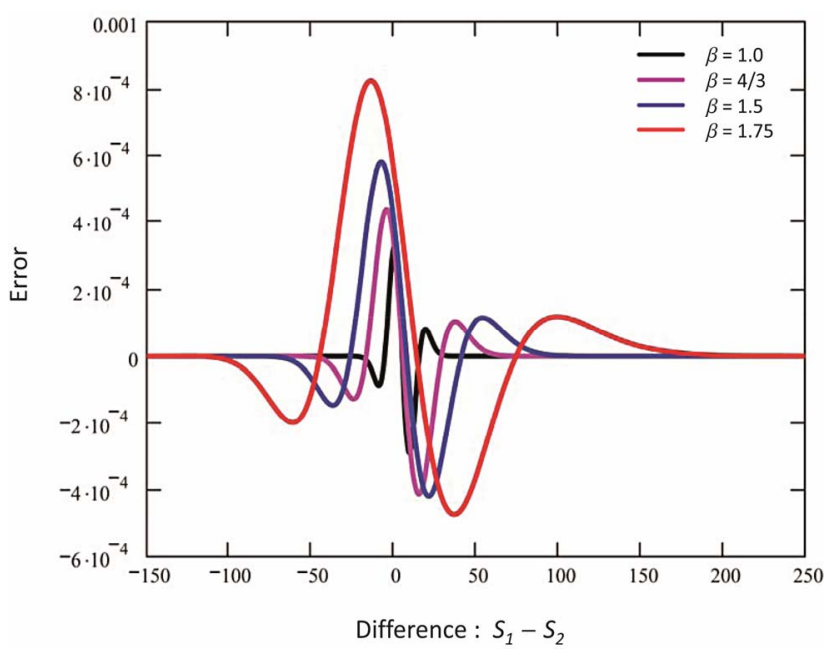

(a)

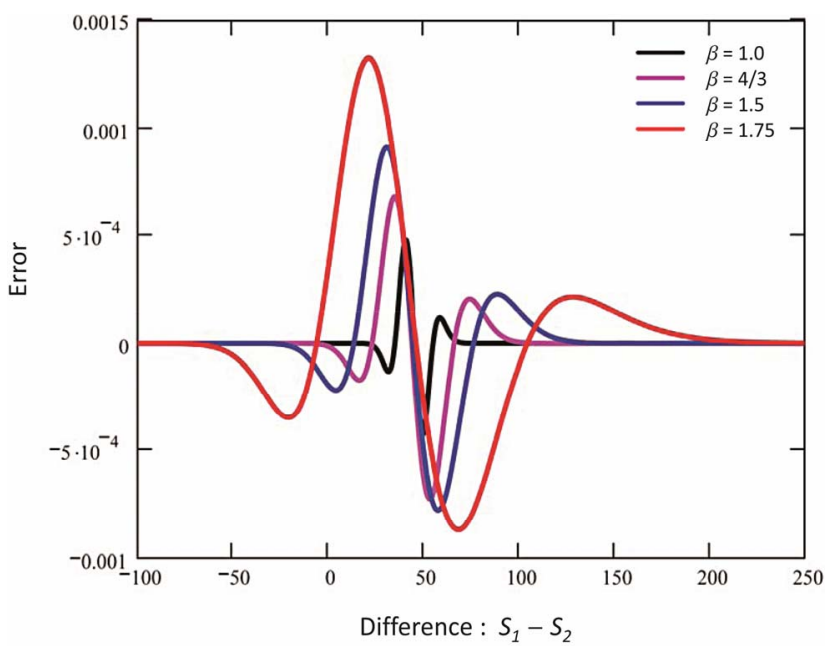

(c)

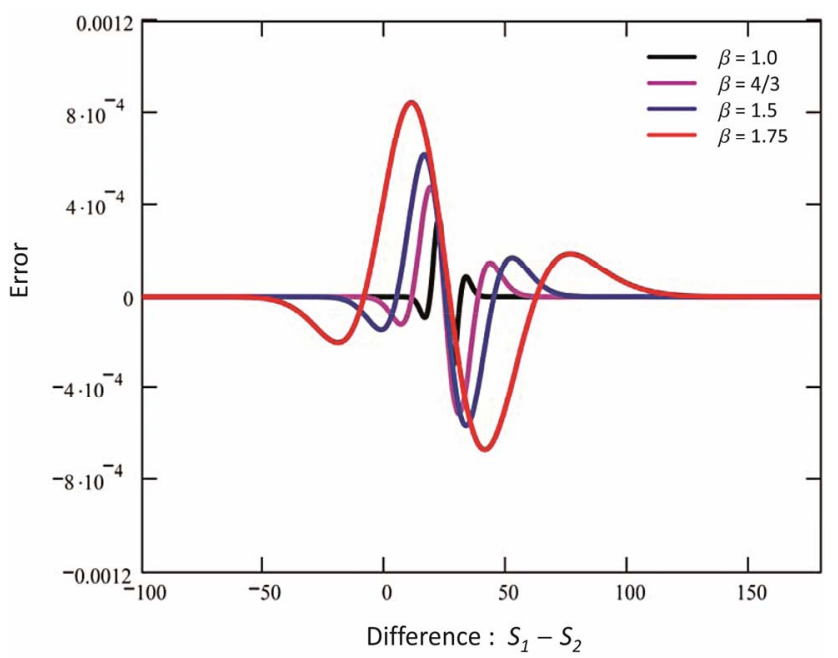

(e)

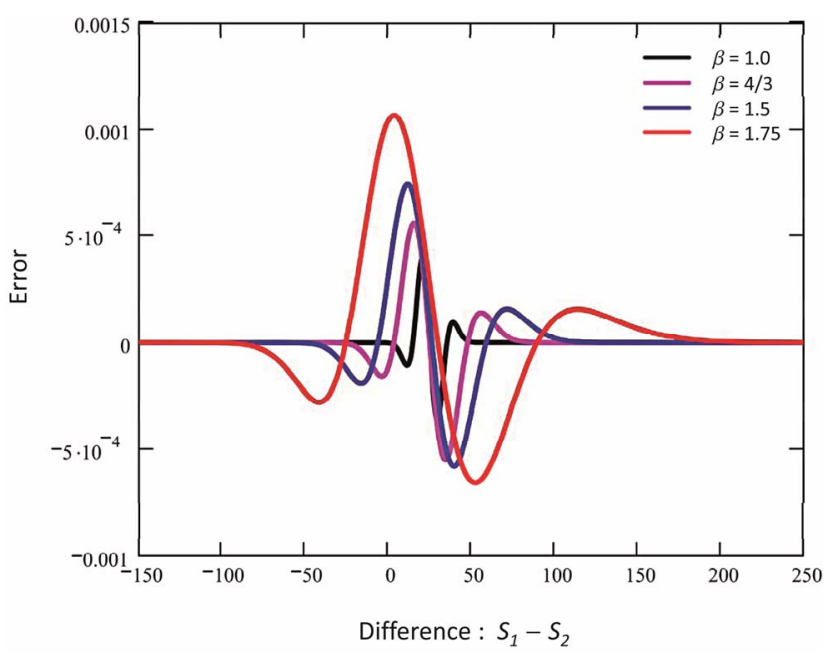

(b)

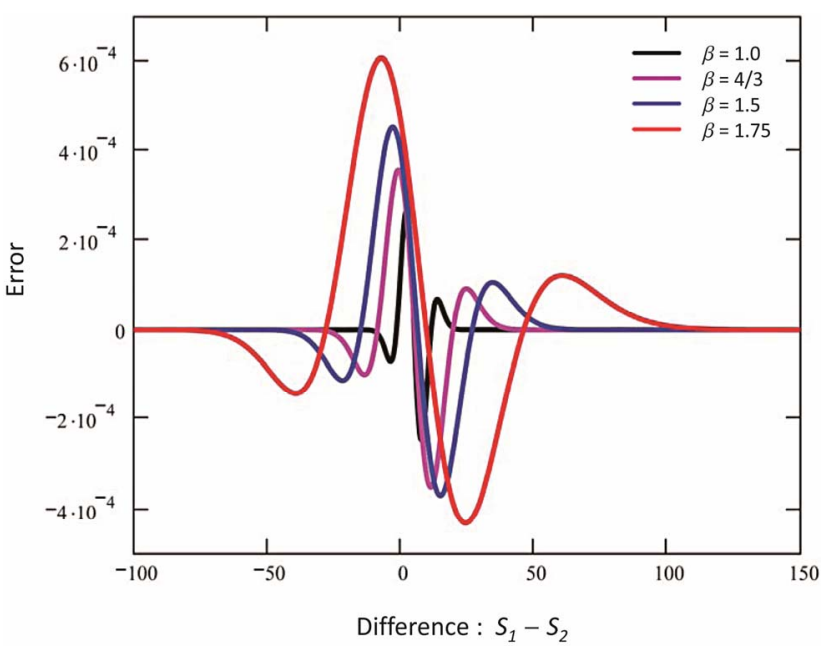

(d)

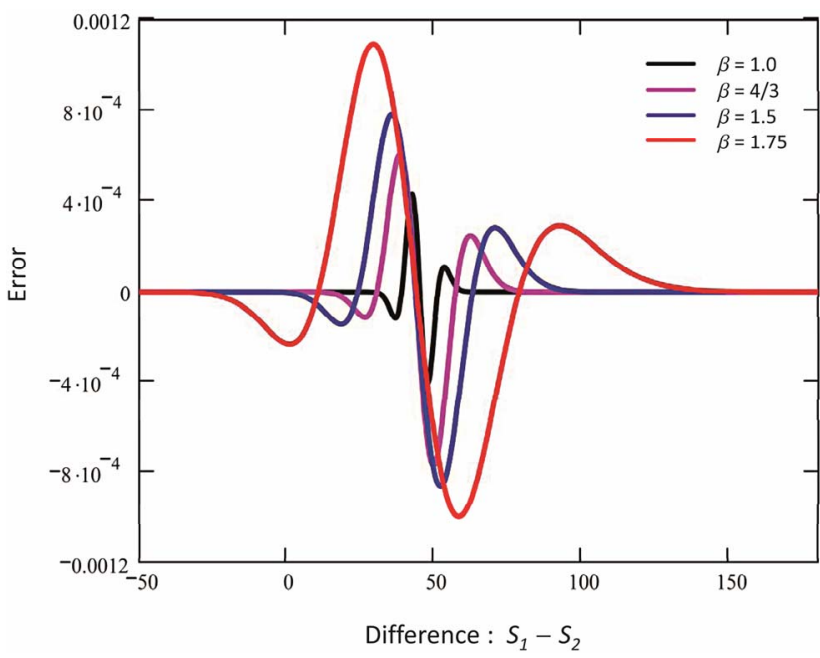

(f)

Figure 4. Error vs. $S_{1}-S_{2}$ : the error is calculated by subtracting the approximate estimate from the exact result. (a) $S_{10}=$ $110, S_{20}=105, \sigma_{1}=0.5$ and $\sigma_{2}=0.3 ;$ (b) $S_{10}=110, S_{20}=85, \sigma_{1}=0.5$ and $\sigma_{2}=0.3$; (c) $S_{10}=110, S_{20}=65, \sigma_{1}=0.5$ and $\sigma_{2}=0.3 ;$ (d) $S_{10}=110, S_{20}=105, \sigma_{1}=0.3$ and $\sigma_{2}=0.2$; (e) $S_{10}=110, S_{20}=85, \sigma_{1}=0.3$ and $\sigma_{2}=0.2$; (f) $S_{10}=110, S_{20}=65, \sigma_{1}=0.3$ and $\sigma_{2}=$ 0.2. 
algebraic sum of $N$ CEV variables with potential applications in pricing multi-asset options.

\section{REFERENCES}

[1] C. F. Lo, "The Sum and Difference of Two Lognormal Random Variables," Journal of Applied Mathematics, Vol. 2012, 2012, Article ID: 838397. http://dx.doi.org/10.1155/2012/838397

[2] L. Fenton, "The Sum of Lognormal Probability Distributions in Scatter Transmission Systems," IRE Transactions on Communications Systems, Vol. 8, No. 1, 1960, pp. 5767. http://dx.doi.org/10.1109/TCOM.1960.1097606

[3] J. I. Naus, "The Distribution of the Logarithm of the Sum of Two Lognormal Variates," Journal of the American Statistical Association, Vol. 64, No. 326, 1969, pp. 655659. http://dx.doi.org/10.1080/01621459.1969.10501004

[4] M. A. Hamdan, "The Logarithm of the Sum of Two Correlated Lognormal Variates," Journal of the American Statistical Association, Vol. 66, No. 333, 1971, pp. 105106. http://dx.doi.org/10.1080/01621459.1971.10482229

[5] C. L. Ho, "Calculating the Mean and Variance of Power Sums with Two Lognormal Components," IEEE Transactions on Vehicular Technology, Vol. 44, No. 4, 1995, pp. 756-762. http://dx.doi.org/10.1109/25.467959

[6] M. A. Milevsky and S. E. Posner, "Asian Options, the Sum of Lognormals, and the Reciprocal Gamma Distribution," Journal of Financial and Quantitative Analysis, Vol. 33, No. 3, 1998, pp. 409-422. http://dx.doi.org/10.2307/2331102

[7] J. Dhaene, M. Denuit, M. J. Goovaerts, R. Kaas and D. Vyncke, "The Concept of Comonotonicity in Actuarial Science and Finance: Applications,” Insurance: Mathematics and Economics, Vol. 31, No. 2, 2002, pp. 133-161. http://dx.doi.org/10.1016/S0167-6687(02)00135-X

[8] R. Carmona and V. Durrleman, "Pricing and Hedging Spread Options,” SIAM Review, Vol. 45, No. 4, 2003, pp. 627-685. http://dx.doi.org/10.1137/S0036144503424798

[9] J. H. Graham, K. Shimizu, J. M. Emlen, D. C. Freeman and J. Merkel, "Growth Models and the Expected Distribution of Fluctuating Asymmetry," Biological Journal of the Linnean Society, Vol. 80, No. 1, 2003, pp. 57-65. http://dx.doi.org/10.1046/j.1095-8312.2003.00220.x

[10] M. Romeo, V. Da Costa and F. Bardon, "Broad Distribution Effects in Sums of Lognormal Random Variables," European Physical Journal B, Vol. 32, No. 4, 2003, pp. 513-525. http://dx.doi.org/10.1140/epjb/e2003-00131-6

[11] D. Dufresne, "The Log-Normal Approximation in Financial and Other Computations," Advances in Applied Probability, Vol. 36, No. 3, 2004, pp. 747-773. http://dx.doi.org/10.1239/aap/1093962232

[12] S. Vanduffel, T. Hoedemakers and J. Dhaene, "Comparing Approximations for Risk Measures of Sums of NonIndependent Lognormal Random Variables,” North American Actuarial Journal, Vol. 9, No. 4, 2005, pp. 71-82.

[13] J. Wu, N. B. Mehta and J. Zhang, "Flexible Lognormal Sum Approximation Method,” Proceedings of IEEE Global Telecommunications Conference (GLOBECOM'05), Vol.
6, 2005, pp. 3413-3417.

[14] A. Kukush and M. Pupashenko, "Bounds for a Sum of Random Variables under a Mixture of Normals," Theory of Stochastic Processes, Vol. 13, No. 29, 2007, pp. 82-97.

[15] X. Gao, H. Xu and D. Ye, "Asymptotic Behavior of Tail Density for Sum of Correlated Lognormal Variables,” International Journal of Mathematics and Mathematical Sciences, Vol. 2009, 2009, Article ID: 630857.

[16] H. F. Trotter, "On the Product of Semi-Groups of Operators," Proceedings of the American Mathematical Society, Vol. 10, No. 4, 1959, pp. 545-551. http://dx.doi.org/10.1090/S0002-9939-1959-0108732-6

[17] N. C. Beaulieu and F. Rajwani, "Highly Accurate Simple Closed-Form Approximations to Lognormal Sum Distributions and Densities," IEEE Communications Letters, Vol. 8, No. 12, 2004, pp. 709-711. http://dx.doi.org/10.1109/LCOMM.2004.837657

[18] C. L. J. Lam and T. Le-Ngoc, "Estimation of Typical Sum of Lognormal Random Variables Using Log-Shifted Gamma Approximation,” IEEE Communications Letters, Vol. 10, No. 4, 2006, pp. 234-235.

http://dx.doi.org/10.1109/LCOMM.2006.1613731

[19] L. Zhao and J. Ding, "Least Squares Approximations to Lognormal Sum Distributions," IEEE Transactions on Vehicular Technology, Vol. 56, No. 2, 2007, pp. 991-997. http://dx.doi.org/10.1109/TVT.2007.891467

[20] N. B. Mehta, J. Wu, A. F. Molisch and J. Zhang, “Approximating a Sum of Random Variables with a Lognormal," IEEE Transactions on Wireless Communications, Vol. 6, No. 7, 2007, pp. 2690-2699. http://dx.doi.org/10.1109/TWC.2007.051000

[21] S. Borovkova, F. J. Permana and H. V. D. Weide, “A Closed Form Approach to the Valuation and Hedging of Basket and Spread Options," The Journal of Derivatives, Vol. 14, No. 4, 2007, pp. 8-24. http://dx.doi.org/10.3905/jod.2007.686420

[22] D. Dufresne, "Sums of Lognormals," Actuarial Research Conference Proceedings, Regina, 14-16 August 2008.

[23] Q. T. Zhang and S. H. Song, "A Systematic Procedure for Accurately Approximating Lognormal-Sum Distributions," IEEE Transactions on Vehicular Technology, Vol. 57, No. 1, 2008, pp. 663-666. http://dx.doi.org/10.1109/TVT.2007.905611

[24] T. R. Hurd and Z. Zhou, "A Fourier Transform Method for Spread Option Pricing,” SIAM Journal of Financial Mathematics, Vol. 1, No. 1, 2010, pp. 142-157. http://dx.doi.org/10.1137/090750421

[25] X. Li, V. D. Chakravarthy and Z. Wu, "A Low-Complexity Approximation to Lognormal Sum Distributions via Transformed Log Skew Normal Distribution,” IEEE Transactions on Vehicular Technology, Vol. 60, No. 8, 2011, pp. 4040-4045.

http://dx.doi.org/10.1109/TVT.2011.2163652

[26] N. C. Beaulieu, “An Extended Limit Theorem for Correlated Lognormal Sums," IEEE Transactions on Communications, Vol. 60, No. 1, 2012, pp. 23-26. http://dx.doi.org/10.1109/TCOMM.2011.091911.110054

[27] J. J. Chang, S. N. Chen and T. P. Wu, “A Note to En- 
hance the BPW Model for the Pricing of Basket and Spread Options,” The Journal of Derivatives, Vol. 19, No. 3, 2012, pp. 77-82. http://dx.doi.org/10.3905/jod.2012.19.3.077

[28] J. C. Cox and S. A. Ross, "The Valuation of Options for Alternative Stochastic Processes," Journal of Financial Economics, Vol. 3, No. 1-2, 1976, pp. 145-166. http://dx.doi.org/10.1016/0304-405X(76)90023-4

[29] J. C. Cox, "The Constant Elasticity of Variance Option Pricing Model,” Journal of Portfolio Management, Vol. 23, 1996, pp. 15-17.

[30] C. F. Lo, P. H. Yuen and C. H. Hui, “Constant Elasticity of Variance Option Pricing Model with Time-Dependent Parameters," International Journal of Theoretical and Applied Finance, Vol. 3, No. 4, 2000, pp. 661-674. http://dx.doi.org/10.1142/S0219024900000814

[31] D. Davydov and V. Linetsky, "The Valuation and Hedging of Barrier and Lookback Option under the CEV Process,” Management Science, Vol. 47, 2001, pp. 949-965. http://dx.doi.org/10.1287/mnsc.47.7.949.9804

[32] J. Detemple and W. D. Tian, "The Valuation of American Options for a Class of Diffusion Processes,” Management Science, Vol. 48, No. 7, 2002, pp. 917-937. http://dx.doi.org/10.1287/mnsc.48.7.917.2815

[33] C. Jones, "The Dynamics of the Stochastic Volatility: Evidence from Underlying and Options Markets," Journal of Econometrics, Vol. 116, No. 1-2, 2003, pp. 181224. http://dx.doi.org/10.1016/S0304-4076(03)00107-6

[34] M. Widdicks, P. W. Duck, A. D. Andricopoulos and D. P. Newton, "The Black-Scholes Equation Revisited: Asymptotic Expansions and Singular Perturbations," Mathematical Finance, Vol. 15, No. 2, 2005, pp. 373-391. http://dx.doi.org/10.1111/j.0960-1627.2005.00224.x

[35] J. Xiao, H. Zhai and C. Qin, "The Constant Elasticity of Variance (CEV) Model and the Legendre TransformDual Solution for Annuity Contracts,” Insurance: Mathematics and Economics, Vol. 40, 2007, pp. 302-310. http://dx.doi.org/10.1016/j.insmatheco.2006.04.007

[36] Y. L. Hsu, T. I. Lin and C. F. Lee, "Constant Elasticity of Variance (CEV) Option Pricing Model: Integration and Detailed Derivation," Mathematics and Computers in Simulation, Vol. 79, No. 1, 2008, pp. 60-71. http://dx.doi.org/10.1016/j.matcom.2007.09.012

[37] R. R. Chen, C. F. Lee and H. H. Lee, "Empirical Performance of the Constant Elasticity Variance Option Pricing Model,” Review of Pacific Basin Financial Markets and
Policies, Vol. 12, No. 2, 2009, pp. 1-41. http://dx.doi.org/10.1142/S0219091509001605

[38] J. Gao, "Optimal Portfolios for DC Pension Plans under a CEV Model,” Insurance: Mathematics and Economics, Vol. 44, No. 3, 2009, pp. 479-490. http://dx.doi.org/10.1016/j.insmatheco.2009.01.005

[39] M. Gu, Y. Yang, S. Li and J. Zhang, “Constant Elasticity of Variance Model for Proportional Reinsurance and Investment Strategies," Insurance: Mathematics and Economics, Vol. 46, No. 3, 2010, pp. 9-18.

[40] X. Lin and Y. Li, "Optimal Reinsurance and Investment for a Jump Diffusion Risk Process under the CEV Model,” North American Actuarial Journal, Vol. 15, No. 3, 2011, pp. 417-431. http://dx.doi.org/10.1080/10920277.2011.10597628

[41] H. Zhao and X. Rong, "Portfolio Selection Problem with Multiple Risky Assets under the Constant Elasticity of Variance Model," Insurance: Mathematics and Economics, Vol. 50, No. 1, 2012, pp. 179-190. http://dx.doi.org/10.1016/j.insmatheco.2011.10.013

[42] J. E. Ingersoll, "Theory of Financial Decision Making,” Rowman \& Littlefield Publishers, Lanham, 1987.

[43] D. R. Cox and H. D. Miller, "Theory of Stochastic Processes," Chapman \& Hall/CRC, London, 2001.

[44] J. Baz and G. Chacko, "Financial Derivatives: Pricing, Applications and Mathematics,” Cambridge University Press, Cambridge, 2009.

[45] G. W. Gardiner, "Handbook of Stochastic Methods for Physics, Chemistry and the Natural Sciences,” Springer, Berlin, 2004.

[46] H. F. Trotter, "Approximation of Semi-Groups of Operators,” Pacific Journal of Mathematic, Vol. 8, No. 4, 1958, pp. 887-919. http://dx.doi.org/10.2140/pjm.1958.8.887

[47] M. Suzuki, "Decomposition Formulas of Exponential Operators and Lie Exponentials with Some Applications to Quantum Mechanics and Statistical Physics,” Journal of Mathematical Physics, Vol. 26, No. 4, 1985, pp. 601612. http://dx.doi.org/10.1063/1.526596

[48] A. N. Drozdov and J. J. Brey, "Operator Expansions in Stochastic Dynamics,” Physical Review E, Vol. 57, No. 2, 1998, pp. 1284-1289. http://dx.doi.org/10.1103/PhysRevE.57.1284

[49] N. Hatano and M. Suzuki, "Finding Exponential Product Formulas of Higher Orders," Lecture Notes in Physics, Vol. 679, 2005, pp. 37-68. 